\title{
Study of Failure Hazard Degree in Large Data Centers
}

${ }^{1}$ Iskandar N. Nasyrov, ${ }^{2}$ Ildar I. Nasyrov, ${ }^{3}$ Rustam I. Nasyrov, ${ }^{4}$ Bulat A. Khairullin

${ }^{1-4}$ Kazan Federal University

Email: ecoseti@yandex.ru

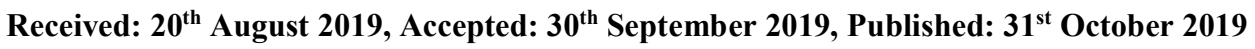

\begin{abstract}
The change in SMART values that occurred during operation, namely, parameters characterizing the reliability of hard magnetic disks in computers, is considered. It is precisely those parameters that are considered critical due to the fact that if their values increase, the probability of failure of information storage devices on hard drives increases. The scientific task of the study is to establish the priority of the specified parameters in failed hard drives from the standpoint of assessing the reliability of information storage devices of various manufacturers. The study analyzed the information storage devices of the brands HGST, Hitachi, Samsung, ST, Toshiba, WDC, operated in the largest commercial data center Backblaze. The analysis revealed the following priority of critical parameters: 5 Reallocated sectors count (number of reassigned sectors), 196 Reallocation event count (number of successful and unsuccessful attempts at the reassignment), 197 Current pending sector count (number of sectors, which are been candidates for the replacement), 1 Read error rate (frequency of errors during reading of data from the disk), 9 Power-on hours (the number of hours, carried out in the switched-on state), 7 Seek error rate (frequency of errors during positioning of the unit of magnetic heads), 10 Spin-up retry count (number of repeated attempts at the spin up of disks to the operating speed if the first attempt was unsuccessful). It is shown that the very existence of the values of the parameters considered depends on the manufacturer of the hard drives. The ranking of information storage devices by the degree of reliability of implementation using the priority parameters identified as a result of the study is proposed.
\end{abstract}

\section{Keywords}

Ranking, Hard Disk, Reliability, Information, Safety, Storage.

\section{Introduction}

To ensure the security of information, it is necessary to copy the data from the unreliable to a new and reliable drive in a timely and complete manner. For this purpose, usually SMART technology is used (self-monitoring, analysis and reporting technology [1]) for internal assessment of the state of the hard disk of the computer, as well as a way to predict the possible failure of it.

The paper considers the change occurred due to the operating time of the values of SMART-parameters characterizing the reliability of hard magnetic disks in computers. The parameters selected are critical in the sense that if their values increase, the probability of failure of the hard disk drives increases. The scientific objective of the study is to establish the priority of these parameters in failed hard drives from the standpoint of assessing the reliability of information storage devices of various manufacturers.

The study analyzed drives of brands HGST, Hitachi, Samsung, ST, Toshiba, WDC, operated in the largest commercial data center Backblaze. The analysis revealed the following priority of critical parameters (in descending order): 5 Reallocated sectors count (number of reassigned sectors),196 Reallocation event count (number of successful and unsuccessful attempts at the reassignment), 197 Current pending sector count (number of sectors, which are been candidates for the replacement), 1 Read error rate (frequency of errors during reading of data from the disk), 9 Poweron hours (the number of hours, carried out in the switched-on state), 7 Seek error rate (frequency of errors during positioning of the unit of magnetic heads), 10 Spin-up retry count (number of repeated attempts at the spin up of disks to the operating speed if the first attempt was unsuccessful).

It is shown that the very presence of the values of the parameters examined depends on the manufacturer of hard disks. The ranking of information storages according to the degree of reliability to carry out with the application of that revealed as a result of a study of parameters priority is proposed.

\section{Methods}

The SMART-parameters given on the Backblaze company site were studied for the analysis of the dependence of parameter values from operation time in the malfunctioned information storages on the hard magnetic disks [2]. It is examined 45 SMART-parameters of 92530 storages of 93 models of 6 trademarks HGST (Hitachi Global Storage Technologies), Hitachi (later HGST), Samsung, ST (Seagate), Toshiba, WDC (Western Digital) for the period from 10 April 2013 to 31 December 2016. It is discovered that at the end of the period being investigated $79.58 \%$ storages continued normally to work, $14.74 \%$ were taken from the operation before the appointed time, $5.68 \%$ malfunctioned. In all the information about the semantic value of more than 80 SMART-parameters is accessible, however, the majority of them are not used by manufacturers. Therefore Backblaze specialists recorded in 2013-2014 only 40 of them, and beginning from 2015 - 45 with the numbers 1-5, 7-13, 15, 22, 183, 184, 187-201, 220, 222-226, 240-242, 250-252, 254, 255 (in 2015 they added 22, 220, 222, 224, 226) (Table 1). 


\begin{tabular}{|c|c|c|c|c|c|c|}
\hline No. & HGST & Hitachi & Samsung & Seagate & Toshiba & WDC \\
\hline 1 & 100.00 & 100.00 & 100.00 & 100.00 & 100.00 & 100.00 \\
\hline 2 & 99.99 & 99.07 & & 1.47 & 99.07 & \\
\hline 3 & 99.99 & 99.07 & 100.00 & 97.58 & 99.07 & 94.04 \\
\hline 4 & 99.99 & 99.07 & 100.00 & 97.58 & 99.07 & 94.04 \\
\hline 5 & 100.00 & 100.00 & 100.00 & 100.00 & 100.00 & 100.00 \\
\hline 7 & 99.99 & 99.07 & 100.00 & 97.58 & 99.44 & 94.04 \\
\hline 8 & 99.99 & 99.07 & 100.00 & 1.47 & 99.44 & \\
\hline 9 & 100.00 & 100.00 & 100.00 & 100.00 & 100.00 & 100.00 \\
\hline 10 & 99.99 & 99.07 & 100.00 & 97.58 & 99.44 & 94.04 \\
\hline 11 & & & 100.00 & 1.47 & & 94.04 \\
\hline 12 & 99.99 & 99.07 & 100.00 & 97.58 & 99.44 & 94.04 \\
\hline 13 & & & 100.00 & & & \\
\hline 15 & & & & 0.00 & & \\
\hline 22 & 0.27 & & & & & \\
\hline 183 & & & 100.00 & 79.62 & & \\
\hline 184 & & & 100.00 & 96.10 & & 0.03 \\
\hline 187 & & & 100.00 & 96.10 & & 0.03 \\
\hline 188 & & & 100.00 & 96.10 & & 0.03 \\
\hline 189 & & & & 96.10 & & \\
\hline 190 & & & 100.00 & 96.10 & & 0.05 \\
\hline 191 & & & & 92.70 & 83.10 & 5.06 \\
\hline 192 & 99.99 & 99.07 & & 92.70 & 99.44 & 92.05 \\
\hline 193 & 99.99 & 99.07 & & 91.23 & 99.44 & 92.05 \\
\hline 194 & 100.00 & 100.00 & 100.00 & 100.00 & 100.00 & 99.07 \\
\hline 195 & & & 100.00 & 26.58 & & \\
\hline 196 & 99.99 & 99.07 & 100.00 & 1.47 & 99.44 & 94.04 \\
\hline 197 & 100.00 & 100.00 & 100.00 & 100.00 & 100.00 & 100.00 \\
\hline 198 & 99.99 & 99.07 & 100.00 & 97.58 & 99.44 & 94.04 \\
\hline 199 & 99.99 & 99.07 & 100.00 & 97.58 & 99.44 & 94.04 \\
\hline 200 & & & 100.00 & 1.47 & & 94.04 \\
\hline 201 & & & 100.00 & & & \\
\hline 220 & & & & & 83.10 & \\
\hline 222 & & & & & 83.10 & \\
\hline 223 & & & & 1.47 & 83.10 & \\
\hline 224 & & & & & 83.10 & \\
\hline 225 & & & & 1.47 & & \\
\hline 226 & & & & & 83.10 & \\
\hline 240 & & & & 95.93 & 83.10 & 2.69 \\
\hline 241 & & & & 95.93 & & 0.65 \\
\hline 242 & & & & 95.93 & & 0.65 \\
\hline 250 & & & & 0.18 & & \\
\hline 251 & & & & 0.18 & & \\
\hline 252 & & & & 0.18 & & \\
\hline 254 & & & & 0.34 & & 0.63 \\
\hline 255 & & & & 0.00 & & \\
\hline
\end{tabular}

Table 1: Manufacturers, who use the Measured by Backblaze Specialists SMART-Parameters, in the

Percentages

As can be seen from Table 1, there is a very limited set of parameters that can be used to diagnose and assess the status of drives of any manufacturers. First of all, it was proposed to use the number of reassigned sectors. But not as a separate alone parameter for assessing reliability, but as a totality of data: current value, average accumulation rate since putting a disk into operation, the instantaneous rate of change in the number of reassigned sectors since the last measurement. A similar combination of average and instantaneous rate of change of parameter values is used by Blackbaze experts [3].

A proof of the priority of the number of reassigned sectors when assessing the state of a hard disk is presented in [4], which shows the results of a study on 100,000 drives in servers around the world, performed by Google.

Additional parameters can be selected in two different ways: relative [5] and absolute values [6].

A similar approach allows you to:

1) Track drives in which the current value is close to the limit level;

2) Keep control of drives that slowly but steadily collapse; 
3) Take emergency measures for drives in which a one-time jump in the number of reassigned sectors causes concern.

\section{Results and Discussion}

Initially, the basic algorithm of standard operations was considered in case of failure of information storage devices and the need to develop an algorithm for assessing and predicting the reliability of storage devices in control systems based on it was substantiated, indicating the included functionality. The first version of the algorithm was intended for oneparameter estimation and prediction of the reliability of information storage devices. In the following, several parameters were already considered.

For a multi-parameter algorithm, it is proposed to apply the experience of Backblaze specialists who use the program Smartmontools [7] to obtain SMART data. Then they add several elements, such as the measurement date, drive model, serial number, capacity, failure indicator and create a line in the daily log for each disk. You can download these log files from the company website. Disk data that is marked as failed on one of the days is not included in the logs from the next day onwards. Sometimes a disk is removed from service even though it has not failed, for example, when the storage module is updated and 1 TB drives are replaced with 4 TB drives. In this case, a 1 TB disk is not marked as bad, but its SMART data is no longer recorded.

On the one hand, adding new repeating elements, such as a drive model, serial number, capacity, seem redundant, on the other hand, they do not allow data to mix or get lost. Therefore, we suggest taking advantage of the Backblaze experience and creating disk state registration files with the indicated additions.

Another problem is the time gap between disk failure and its replacement. The fact is that the sign of failure (one - if failed, zero - in other cases) is set manually after replacing the disk in the module. And before that, it can stand there for several days. However, data from it will not be recorded (it does not work after all), although the sign will indicate that it is still operational. As a result of this, you also have to manually fill in the empty spaces with the latest relevant data or change the date of failure. This order is necessary to provide the ability to process data with standard programs such as Excel.

In the analysis, it is proposed to take into account the priority for users of both reliability data and the type of hard drive failures. The highest priority is a group of parameters related to the state of the memory space: 5 Reallocated sectors count (the number of reassigned sectors), 196 Reallocation event count (the number of successful and unsuccessful reassignment attempts), 197 Current pending sector count (the number of sectors that are candidates for replacement), 1 Read error rate (frequency of errors during reading of data from the disk). These parameters are always available for all drives of all types of almost all manufacturers (196 is not available for Samsung and Seagate hard drives (trademark $\mathrm{ST})$ ).

The second highest priority is to apply the standard definition of reliability as the time between failures, which corresponds to the always available parameter 9 Power-on hours (the number of hours spent on switched).

The third priority is a group of parameters related to the positioning of the read/write head: 7 Seek error rate (frequency of errors when positioning the block of magnetic heads), 2 Throughput performance (total disk performance), 8 Seek time performance (average performance of the magnetic head positioning operation). Head positioning errors are the main reason for reassigning sectors and subsequent ST drive failure. However, parameter 7 has zero values or is absent for Samsung and Toshiba drives (possibly due to small statistics), and parameters 2 and 8 are not present for Samsung, ST, WDC drives. Therefore, in the future, only parameter 7 is considered as an independent indicator of reliability.

The fourth priority is a group of parameters related to the mechanics for rotating hard drives: 10 Spin-up retry count (the number of retries to spin up the drives to operating speed if the first attempt was unsuccessful), 3 Spin-up time (time to spin up the disk pack from standstill to operating speed), 4 Start / stop count (total number of spindle start / stop cycles), 12 Device power cycle count (number of complete disk on / off cycles), 192 Power-off retract count (number of shutdown or emergency cycles failures (power on / off)), 193 Load / unload cycle (the number of magnetic heads blocks displacement cycles in the parking zone/in the operating position). Parameters 4, 12, 192, 193 are cumulative and at large values those changes that are due to the positioning of the heads become invisible. And when analyzing parameter 3, its deviation from the norm, which is unknown, is important. Therefore, for further consideration, only parameter 10 can be left.

In practical software implementation of the algorithm, information on the parameters for the last date is read from the SMART database. The received data is sorted in descending order. Then the data is marked graphically in color for visualization of the drive status. For color gradation, the boundaries of hazard levels are used.

As noted above, there have been cases where failed drives have not been replaced for several days. These are the hard drives of the HGST HMS5C4040BLE640 models (1 pc.), HGST HMS5C4040ALE640 (5 pcs.), ST4000DM000 (3 pcs.), all with a capacity of 4 TB. At the same time, the parameter values were not fixed, although signs of failure showed that the drives were operational. Cases were also revealed when during the work on the WDC WD10EADS model drives with a capacity of 1 TB (2 pcs.) and ST4000DM000 with a capacity of 4 TB (52 pcs.) data was not indicated on one of the days. Also, a case was detected of a failure of parameter fixation two days before the complete failure of the hard drive of the ST4000DM000 model with a capacity of $4 \mathrm{~TB}$, in which parameter 1 was empty, and the rest had random values. The WDC WD1600AAJS model drive with a capacity of $0.16 \mathrm{~TB}$ did not have data on parameter 194 from the very beginning. Therefore, it is necessary to organize a check for such situations and prepare the corresponding corrective actions. 
Given the fact that sectors were reassigned by entire tracks (multiple of 8) [8], it is proposed to apply the following color allocation of the drive failure hazard levels:

Colorless (green) - with a zero or missing parameter value;

Yellow - if the parameter value is 1, i.e. nonzero;

Orange - if the parameter value is greater than 1 and less than or equal to 8 , this will allow detecting errors in the positioning of the heads;

Red - if the parameter value is greater than 8, which means a continuing deterioration of the drive;

Black fragment with a red background - if the drive has already failed, but not yet been replaced, as well as in the event of a failure in which at least one of the usually permanently filled parameters 1, 5, 9, 194, 197 is empty.

\section{Summary}

Therefore, based on the meaning and availability of parameter values when ranking drives according to the degree of failure hazard, you need to use sorting first by parameters 5, 196, 197 and 1, then by 9, then by 7 and 10 .

The software algorithm for ranking information storage devices according to the degree of danger of failure should include three blocks:

1) Reading information from the SMART database;

2) Sorting drives according to parameter values according to the priority of the type of danger of reducing reliability;

3) Correlation of drives by failure hazard levels with the corresponding color marking.

The scientific novelty of the results is that based on the identified priorities of the reliability parameters, the values of which are available for hard magnetic disks of all manufacturers, it is possible to develop an algorithm for ranking information storage devices according to the degree of failure hazard.

\section{Conclusions}

Another argument in support of the use of these parameters is that to characterize the state of the memory space, the same parameters are used with the same meaning in solid-state storage devices [9, 10]. Naturally, the positioning parameters of the write/read heads or disk rotation mechanisms in solid-state drives are not used, although the numbering remains the same. Therefore, in the future, when large data centers move to store information in drives of the indicated type, no additional changes other than the exclusion of parameters 7 and 10 will have to be made to the developed algorithm.

Backblaze experts report the results of their research at conferences [11, 12], however, topics of algorithms for ranking information storage devices by the degree of failure hazard do not affect. Therefore, the developed algorithm has scientific novelty and allows the individual assessment of the reliability of information storage devices to be solved using the identified priority parameters.

\section{Acknowledgments}

The work is performed according to the Russian Government Program of Competitive Growth of Kazan Federal University.

\section{References}

[1] ATA S.M.A.R.T. From Wikipedia, the free encyclopedia. URL: https://en.wikipedia.org/wiki/Smart Retrieved 03/24/ 2019.

[2] Hard Drive Data and Stats / Backblaze. URL: https://www.backblaze.com/b2/hard-drive-test-data.html. Retrieved $03 / 24 / 2019$.

[3] A. Klein. Hard Drive Reliability Stats for Q1 2015. URL: https://www.backblaze.com/blog/hard-drive-reliabilityq1-2015/. Retrieved 03/24/ 2019.

[4] E. Pinheiro, Weber W.-D., Barroso L.A. Failure Trends in a Large Disk Drive Population // The Proceedings of the 5th USENIX Conference on File and Storage Technologies (FAST'07). San Jose, California, USA, February. pp.13-16, 2007.

URL: http://static.googleusercontent.com/external_content/untrusted_dlcp/research.google.com/ru//archive/disk_failures. pdf.

[5] I.N. Nasyrov, I.I. Nasyrov, R.L. Nasyrov, B.A. Khairullin. Data mining for information storage reliability assessment by relative values // International Journal of Engineering and Technology (UAE). 2018. Vol.7, Is.4.7 Special Issue 7. pp. 204-208. URL: https://www.sciencepubco.com/index.php/ijet/article/view/20545.

[6] I.N. Nasyrov, I.I. Nasyrov, R.L. Nasyrov, B.A. Khairullin. Parameters selection for information storage reliability assessment and prediction by absolute values // Journal of Advanced Research in Dynamical and Control Systems. 2018. Vol.7, Is.4.7 Special Issue $7 . \quad$ pp. 2248-2254. URL: http://jardcs.org/backissues/abstract.php?archiveid=5363.

[7] smartmontools/Edgewall Software. URL: https://www.smartmontools.org. Retrieved 03/24/ 2019.

[8] I.N. Nasyrov, I.I. Nasyrov, R.L. Nasyrov, B.A. Khairullin. Dependence of reallocated sectors count on HDD power-on time// International Journal of Engineering and Technology (UAE). 2018. Vol.7, Is.4.7 Special Issue 7. pp. 200-203. URL: https://www.sciencepubco.com/index.php/ijet/article/view/20544. 
[9] SMART Attribute Details / Kingston Technology Corporation. URL: https://drive.google.com/file/d/0B2RTg5K2_LNEZWpER1BjQ3BaM00/view. Retrieved 24/03/ 2019.

[10] Technical note: Client SATA SSD SMART Attribute Reference / Micron Technology, Inc. URL: https://drive.google.com/file/d/0B2RTg5K2_LNEZWpER1BjQ3BaM00/view. Retrieved 24/03/ 2019.

[11] A. Klein. Behind the Curtain of Backblaze Hard Drive Stats // 33rd International Conference on Massive Storage Systems and Technology (MSST 2017). Santa Clara, CA, USA, May 15-19, 2017. URL: http://storageconference.us/2017/index.html. Retrieved 24/03/ 2019.

[12] A. Klein. What Can We Learn From 100,000 Spinning Hard Drives? // Storage Developer Conference (SDC 2017). Santa Clara, CA, USA, September 11-14, 2017. URL: https://sniasdc17.pathable.com/meetings/549460. Retrieved 24/03/ 2019. 